\title{
Mitochondrial Damage and Drp1 Overexpression in Rifampicin- and Isoniazid-induced Liver Injury Cell Model
}

\author{
Fangfang $\mathrm{Li}^{\# 2}$, Juan $\mathrm{Zhou}^{\# 3}$, Yi Li ${ }^{4}$, Kewei Sun ${ }^{5}$ and Jun Chen ${ }^{1 *}$ \\ ${ }^{1}$ Department of Liver Diseases, Third Hospital of Shenzhen, Shenzhen, Guangdong, China; ${ }^{2}$ Department of Digestive Diseases, \\ First Hospital of Chenzhou, Chenzhou, Hunan, China; ${ }^{3}$ Department of Infectious Diseases, The Affiliated Zhuzhou Hospital \\ Xiangya Medical College CSU, Zhuzhou, Hunan, China; ${ }^{4}$ Liver Diseases Center, Department of Infectious Diseases, The Second \\ Xiangya Hospital, Central South University, Changsha, Hunan, China; ${ }^{5}$ Department of Infectious Diseases, First Hospital of Hunan \\ University of Chinese Medicine, Changsha, Hunan, China
}

\begin{abstract}
Background and Aims: Rifampicin (RFP) and isoniazid (INH) are widely used as anti-tuberculosis agents. However, the mechanisms underlying the involvement of reactive oxygen species and mitochondria in RFP- and INH-related hepatotoxicity have not been established yet. This study aimed to observe the intracellular mechanisms leading to mitochondrial dysfunction and morphological changes in RFPand INH-induced hepatocyte injury. Methods: Cell injury, changes in mitochondrial function, and expression and activation of dynamin related protein 1 (Drp1), known as the main protein for mitochondrial fission, were analyzed in cultured QSG7701 cells exposed to RFP and INH. Results: INH and RFP treatment induced pronounced hepatocyte injury and increased cell death. In the similar context of aspartate aminotransferase elevation and adenosine triphosphate synthesis decrease, changes in mitochondrial membrane permeability and reactive oxygen species in hepatocytes induced by RFP were significantly different from those induced by INH $(p<0.05)$. Particularly, we observed the overactivation and mitochondrial translocation of Drp1 in RFP-induced cell injury, which was not occurred with exposure to INH. Conclusions: RFP-induced hepatotoxicity may be closely related to mitochondrial dysfunction and Drp1mediated mitochondrial fission.
\end{abstract}

Citation of this article: Li F, Zhou J, Li Y, Sun K, Chen J. Mitochondrial damage and Drp1 overexpression in rifampicinand isoniazid-induced liver injury cell model. J Clin Transl Hepatol 2019;7(1):40-45. doi: 10.14218/JCTH.2018.00052.

\section{Introduction}

According to the World Health Organization, tuberculosis remains the leading public health concern worldwide. ${ }^{1}$ For

Keywords: Anti-tuberculosis drug-induced liver injury; Drp1; Mitochondrial dysfunction; Mitochondrial fission.

Abbreviations: AST, aspartate aminotransferase; ATP, adenosine triphosphate DILI, drug-induced liver injury; Drp1, dynamin related protein 1; INH, isoniazid RFP, rifampicin; ROS, reactive oxygen species; SPEV, swine embryonal kidney cells; SWFI, sterile water for injection.

Received: 20 September 2018; Revised: 17 December 2018; Accepted: 29 December 2018

${ }^{\#}$ These two authors contributed equally to this work.

*Correspondence to: Jun Chen, Department of Liver Diseases, Third Hospital of Shenzhen, Shenzhen, Guangdong 311115, China. E-mail: chenjun66@csu.edu.cn many decades, rifampicin (RFP) and isoniazid (INH) have remained the most commonly used first-line anti-tuberculosis drugs, but have been known to be hepatotoxic and have already attracted immense attention. The clinical manifestation of hepatotoxicity occurs as an elevated transaminase level, leading to acute liver failure with a high mortality. Liver injury induced by anti-tuberculosis drugs is one of the leading causes of acute liver failure worldwide; ${ }^{2}$ however, the related pathophysiological processes are still obscure.

A previous study reported that oxidative stress resulting from reactive oxygen species (ROS) overproduction is the major pathogenesis of apoptosis in INH- and RFP-induced hepatotoxicity in mice. ${ }^{3}$ Simultaneously, human growth hormone could also remarkably alleviate oxidative stress damage and RFP- and INH-induced apoptosis in mice, suggesting that ROS might fundamentally be crucial in the pathogenesis of RFP- and INH-induced hepatotoxicity through the deterioration of cellular functions. ${ }^{4}$ Furthermore, vitamin $\mathrm{E}$, as an antioxidant, showed a significantly hepatoprotective effect compared with the oral administration of vitamin $E$ and therapeutic doses of RFP. ${ }^{5}$ Indeed, superoxide generation in urine in an experimental drug-induced liver injury (DILI) group conspicuously increased, as compared with that in the non-DILI group, further indicating the involvement of oxidative stress in hepatotoxic pathology through excessive ROS production induced by the INH, RFP, pyrazinamide, and ethambutol regimen (known as the HRZE regimen). ${ }^{6}$

Mitochondria are one of the important sites of ROS production. Knockdown of fusion proteins, such as OPA1 or MFNs, induce mitochondrial fragmentation accompanied by an increase in the ROS level. However, knockdown of fission proteins (Fis1 and dynamin related protein 1 (Drp1)), indicating mitochondrial fusion, had no influence on ROS production in human bronchial epithelial cells. ${ }^{7}$ Deranged dynamics of mitochondrial fission and fusion is often associated with mitochondrial dysfunction and is a characteristic feature of various disorders. RFP was found to induce mitochondrial fragmentation and a spherical shape of swine embryonal kidney cells (commonly known as SPEV) cells. ${ }^{8}$ However, the role of Drp1, which is one of the main players in these processes, has not been investigated in RFP-associated hepatotoxicity. ${ }^{9,10}$

Drp1 translocation from the cytoplasm to the mitochondria, which organizes the oligomer ring around the mitochondria, is an initial step during mitochondrial fission. In addition, the cytoplasmic concentration of Drp1 could increase the size of the oligomeric form, which might be an indicator of 
mitochondrial fission. ${ }^{11}$ Hence, attempts were made to test the hypothesis that RFP or INH and its metabolites, which are closely related to liver injury manifesting as apoptosis and proliferation, have a substantial influence on the expression and activity of Drp1.

\section{Methods}

\section{Cell culture and treatment}

Experiments were performed on the cells of human hepatocyte line QSG-7701 in this study. The cells were cultured under standard conditions in Dulbecco's modified Eagle's medium plus $10 \%$ fetal bovine serum (Gibco, USA) and $100 \mathrm{U} / \mathrm{mL}$ penicillin and $100 \mathrm{mg} / \mathrm{mL}$ streptomycin (Gibco) at $37^{\circ} \mathrm{C}$ in a humidified incubator with a $5 \% \mathrm{CO}_{2}$ atmosphere. They were seeded at a density of $2 \times 10^{6}$ cells/well in sixwell plates for $24 \mathrm{~h}$ before stimulation and then exposed to INH (0.24 M, dissolved with sterile water for injection $(\mathrm{SWFI}))$, RFP (0.18 mM, dissolved with SWFI), and SWFI for $72 \mathrm{~h}$.

\section{Cell biochemical analysis}

The level of DILI was evaluated by measuring aspartate aminotransferase (AST) and alanine aminotransferase levels in the culture supernatant according to a manufacturer's protocol.

\section{Cell viability analysis}

Apoptosis and necrosis of QSG7701 cells were evaluated using an Annexin V/propidium iodide kit (BD Biosciences, USA), according to the manufacturer's protocol. After $72 \mathrm{~h}$ of exposure to stimuli, the cells were collected by treating them with trypsin-EDTA-free medium (0.25\%) (Sigma, USA), centrifuged at $2000 \mathrm{rpm}$, washed with pre-cold phosphatebuffered saline, and suspended in $300 \mu \mathrm{L}$ of the buffer. The single-cell suspension was stained with $5 \mu \mathrm{L}$ of Annexin $\mathrm{V} /$ fluorescein 5-isothiocyanate for $15 \mathrm{~m}$ at room temperature, in the dark. Thereafter, the cells were incubated with $5 \mu \mathrm{L}$ of propidium iodide for $5 \mathrm{~m}$ before analysis by flow cytometry. Data were analyzed using FlowJo 9.3.2 software (Tree Star, USA).

\section{Determination of caspase-3 activity}

Caspase- 3 activity was determined using the luminescent Caspase-Glo 3 Assay (Promega, Switzerland), which was performed according to the manufacturer's protocol.

\section{Determination of mitochondrial membrane potential}

QSG7701 cells were exposed to drugs and phosphatebuffered saline for $72 \mathrm{~h}$ and labeled with MitoTracker Red CMXRos (Invitrogen, USA), according to the manufacturer's protocol. The culture media were removed from the plates and treated with the pre-warmed $\left(37^{\circ} \mathrm{C}\right)$ staining solution containing a $100 \mathrm{nM}$ MitoTracker probe. After incubation for $20 \mathrm{~m}$ at $37^{\circ} \mathrm{C}$ in the incubation box, the staining solution was replaced with fresh pre-warmed phosphate-buffered saline, and the cells were observed using a fluorescence microscope.

\section{Determination of ROS}

MitoSOX Red (Invitrogen) was used as the mitochondrial superoxide indicator. After $72 \mathrm{~h}$ of exposure to stimuli, QSG7701 cells were washed with phosphate-buffered saline and incubated with $1.0-2.0 \mathrm{~mL}$ of $5 \mu \mathrm{M}$ MitoSOX reagent for $10 \mathrm{~m}$ at $37^{\circ} \mathrm{C}$, away from light. Images were then taken using a fluorescence microscope.

\section{Determination of adenosine triphosphate (ATP) levels}

The cellular ATP concentration was measured using a Molecular Probes ATP Determination Kit (Invitrogen), according to the manufacturer's protocol. In brief, the cells of each group were lysed in $50 \mu \mathrm{L}$ of ATP assay buffer, and total ATP levels were determined at $560 \mathrm{~nm}$ using a microplate reader.

\section{Western blot analysis}

The western blot analysis of DRP1 was performed with rabbit anti-human DRP1 antibodies (Cell Signaling, USA) and secondary antibodies (Cell Signaling). Whole-cell lysates were resolved using sodium dodecyl sulfate-polyacrylamide gel, and proteins were transferred to membranes followed by an immunoblot with the appropriate primary (dilution 1:500-1000) and secondary antibodies (dilution 1:5000$10,000)$. Visualization by chemiluminescence was used for presentation.

\section{Immunofluorescence analysis}

After treatments, the cells were washed with phosphatebuffered saline and fixed with $4 \%$ paraformaldehyde for $20 \mathrm{~m}$. They were then permeabilized with $0.25 \%$ Triton $\mathrm{X}-100$ for $10 \mathrm{~m}$, blocked with $5 \%$ bovine serum albumin for $30 \mathrm{~m}$, incubated with primary antibody mouse monoclonal anti-TOM20 (BD, USA) and rabbit monoclonal anti-phosphoDrp $1^{\mathrm{S} 616}$ (Cell Signaling) overnight at $4^{\circ} \mathrm{C}$. Then, they were washed and incubated with secondary antibody (Alexa Fluor 488 donkey anti-rabbit immunoglobulin G (IgG) and Alexa Fluor 594 goat anti-mouse IgG $(\mathrm{H}+\mathrm{L})$ antibody; Molecular Probes) for $1 \mathrm{~h}$ at $37^{\circ} \mathrm{C}$. Further, they were washed with phosphate-buffered saline. The nuclei were stained with DAPI (Invitrogen). Images were visualized under a $100 \times$ objective Olympus FluoView1000 confocal microscope using anti-p-drp1 (s616) (1:1000 dilution, green) and anti-Tom20 (a marker of mitochondria; 1:1000 dilution, red) antibodies. The images were quantified with the ImageJ software.

\section{Statistical analysis}

The unpaired $t$-test (Microsoft Excel 2016) was used to compare the results among treatments. Significance was defined a priori as a $p$ value of $<0.05$.

\section{Results}

\section{INH- and RFP-induced hepatocyte injury}

The cell injury was assessed by evaluating cell death and transaminase levels in vitro. Compared with the control, the incubation of QSG7701 cells with RFP and INH induced a significant increase in alanine aminotransferase and AST levels in the supernatant after 3 -day exposure ( $p<0.05$; Fig. $1 \mathrm{~A})$. 
The flow cytometry analysis with Annexin V/propidium iodide staining showed that both INH and RFP could strongly induce apoptosis and necrosis of QSG7701 cells compared with the control ( $p<0.05$; Fig. 1B and 1C), especially under the stimulation of RFP. Moreover, RFP had an even greater effect on apoptosis compared with INH ( $p<0.05$; Fig. 1C and 1D). It was shown by a quantitative analysis of caspase- 3 production that QSG7701 cells markedly increased production under the influence of RFP compared with other groups $(p<0.05$; Fig. 1E). These results indicated that cell death due to RFP was mainly through caspase-dependent apoptosis; other mechanisms might exist for INH-induced cell damage.

\section{INH- and RFP-induced mitochondrial dysfunction}

The mitochondrial membrane potential and ROS and ATP production were detected to determine the changes in mitochondrial function so as to further characterize the impact of RFP and INH on mitochondria. After incubating the cells with specific drugs for $72 \mathrm{~h}$, the mitochondrial membrane potential was found to be substantially lower in RFP-treated cells compared with INH-treated cells and control group (Fig. 2A). A bright luminescence of ROS in mitochondria was observed, in particular for RFP-treated cells, compared with the control group (Fig. 2B). Simultaneously, the decrease in ATP production in the cells under the influence of RFP and INH was remarkably different from that in the control group $(p<0.05$; Fig. 2C and 2D). Also, notably, the effects of RFP on ATP synthesis were similar to those of INH. However, the impact of RFP on ROS production and mitochondrial membrane permeability was significantly stronger than that of INH. These data showed that INH, especially RFP, induced severe mitochondrial dysfunction in QSG7701 cells.

\section{Expression and translocation of DRP1 to mitochondria under the influence of drugs}

The western blot analysis showed that RFP dramatically augmented the concentration of Drp1 in QSG7701 cells compared with INH-treated cells and control group (Fig. 3A). Confocal microscopy showed that DRP1 was translocated to mitochondria. Serine 616 (S616) phosphorylation of human Drp1 is the activated form of Drp1 in cells that mediates mitochondrial fragmentation. Consequently, the phosphorylation of Drp1 $1^{\mathrm{S} 616}$ ( $\mathrm{p}$-Drp1)/Tom20 co-localization was analyzed. The findings consistently revealed that $\mathrm{p}$-Drp1 was more frequently localized on mitochondria in QSG7701 cells exposed to RFP compared with INH-treated cells and control group (Fig. 3B). These observations indicated that RFP had a drastic influence on mitochondrial fission resulting in damage; INH had no such effects.

\section{Discussion}

The observed effects included reduced mitochondrial membrane integrity, excessive production of ROS, and considerably decreased ATP production when QSG7701 cells were exposed to RFP and INH, indicating that mitochondria were the target of the toxic effects of drugs. The RFP-mediated effects on mitochondria were also shown to be related to the activity of Drp1, a GTPase predominantly controlling mitochondrial fission. Necrosis due to RFP was found to be closely associated with apoptosis, which was confirmed by the observed overexpression of caspase-3. Meanwhile, INH-induced cell death might partially be caused via other mechanisms independent of the caspase- 3 pathway and insensibly associated with ROS and Drp1 abnormalities
A

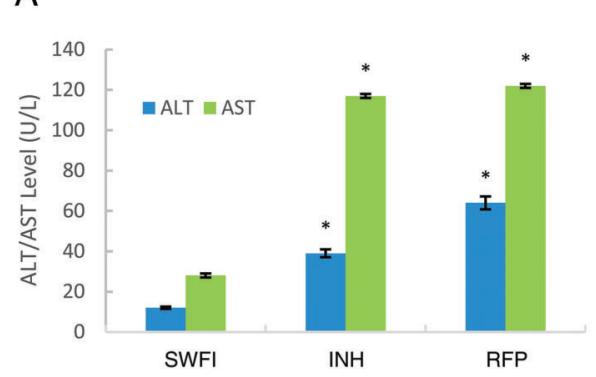

B

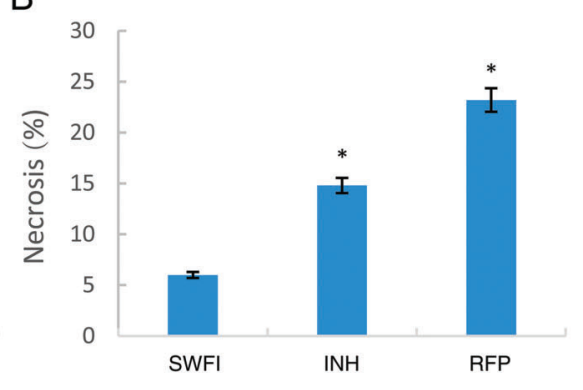

C

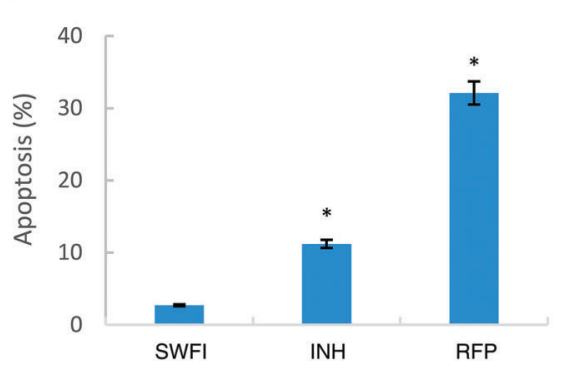

$\mathrm{E}$

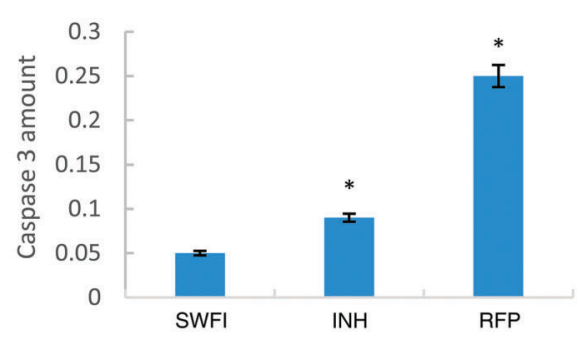

Fig. 1. Hepatotoxicity induced by RFP- and INH-stimulated QSG7701 cells. (A) AST and alanine aminotransferase levels in cell supernatant $72 \mathrm{~h}$ after stimulation. After $72 \mathrm{~h}, p<0.05$ for $0.24 \mathrm{mM}$ RFP and $18 \mathrm{mM}$ INH versus SWFI as blank control. Data represent the results of one of three similar experiments. (B) After $72 \mathrm{~h}, p<0.05$ for RFP and INH versus phosphate-buffered saline, when comparing the proportion of necrotic cells. (C) After $72 \mathrm{~h}, p<0.05$ for RFP and INH versus SWFI, when comparing the proportion of apoptotic cells. (D) Annexin V/propidium iodide staining was assessed in every group by flow cytometry. (E) Caspase-3 levels in cells $72 \mathrm{~h}$ after addition of RFP, $\mathrm{INH}$, or control, determined using luminescent Caspase-Glo 3 Assay $(p<0.05$ for drugs vs. control). 
A
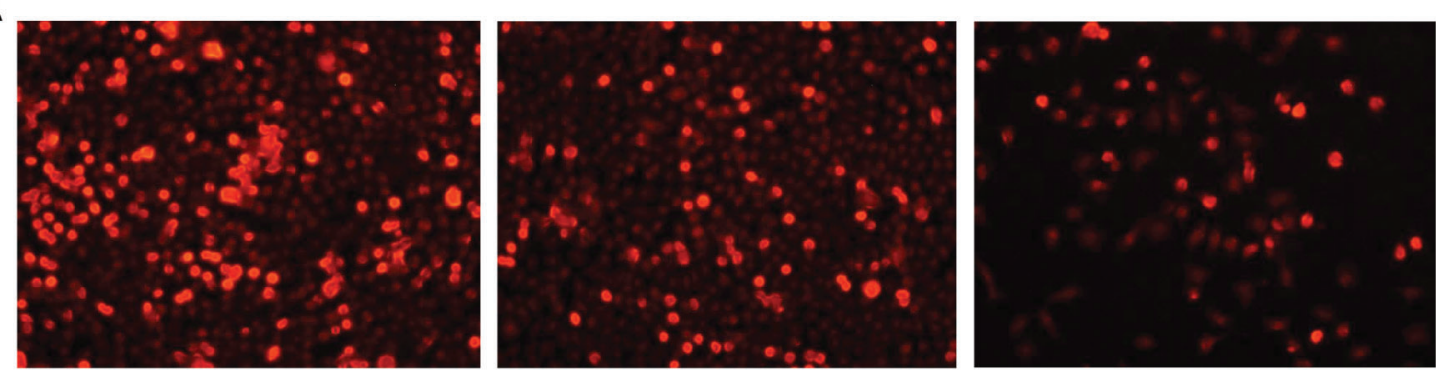

B
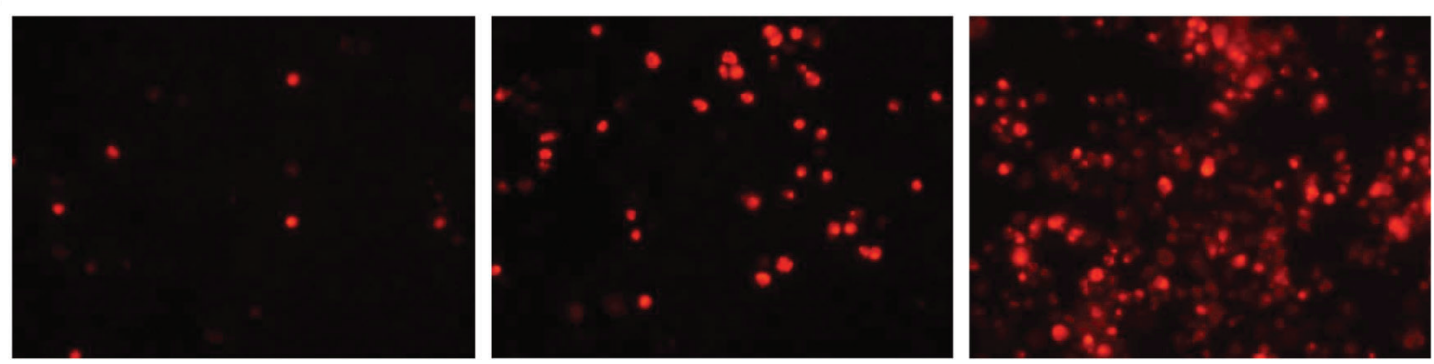

C

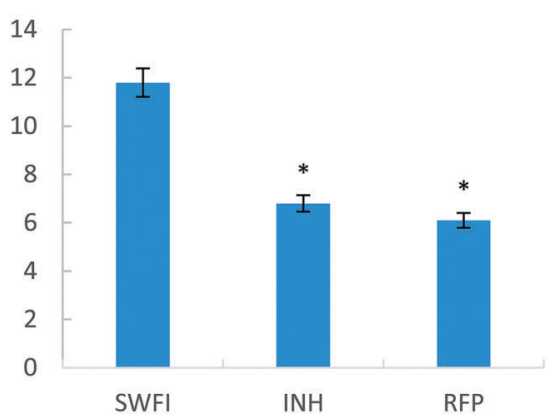

D

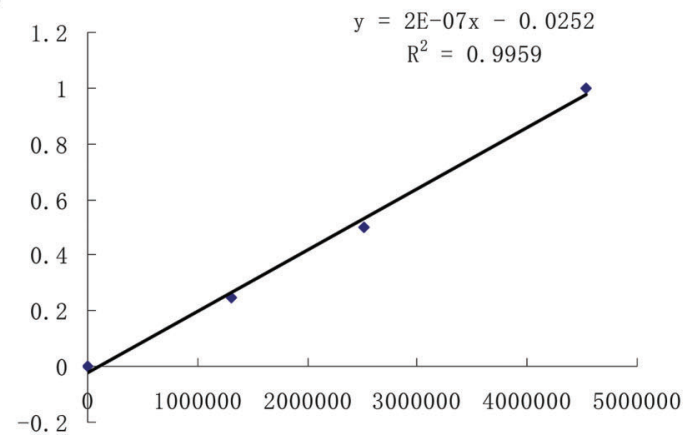

Fig. 2. Toxicity effect on mitochondria in RFP- and INH-stimulated QSG7701 cells. (A) Integrity of mitochondrial membrane potential was evaluated by staining cells with MitoTracker Red CMXRos after 72-h exposure to RFP and INH compared with the control group. (B) Inducible ROS production in QSG7701 cells for each group was assessed using a MitoSOX Red mitochondrial superoxide indicator by immunofluorescence microscopy $(200 \times)$. (C and D) Cellular ATP production for each group ( $<<0.05$ for drugs vs. control).

when the extent of hepatocyte injury is similar to that with RFP. In comparison to INH, the most characteristic features of the RFP-induced liver cell injury model were the activation of DRP1 and translocation to mitochondria, which imply Drp1 may involve DILI caused by RFP. ${ }^{11,12}$

In the present study, hepatocyte injury after RFP and INH stimulation was evaluated by traditional clinical chemistry analyses and flow cytometry. The incubation of cells with $18 \mathrm{mM}$ INH and $0.24 \mathrm{mM}$ RFP in vitro led to remarkable hepatocyte injury manifestations, including significantly increased AST. When exposed to RFP, there was a remarkable increase of ROS and mitochondrial permeability. In contrast, the effect of INH was similar to that of RFP only in terms of ATP reduction, implying that the mechanism of INH-induced mitochondrial dysfunction might be different. Although INH-induced hepatotoxicity was also caused by mitochondrial stress, ${ }^{13}$ similar mitochondrial fragments were also observed in HepG2 cells under stimulation of INH. In contrast, regardless of Drp1 overexpression, the INH-induced mitochondrial fragments are caused by the inhibition of fusion protein MFN2 expression. ${ }^{14}$ Moreover, knockdown of fusion proteins, such as OPA1 and MFNs, apparently leads to mitochondrial fragmentation, simultaneously promoting increased ROS. ${ }^{7}$ In conclusion, it is reasonable to believe that the mechanisms are completely different between RFP and INH inducing mitochondrial fragmentation.

Mounting evidence shows that the toxic effect of RFP is closely related to the excessive production of ROS and mitochondrial dysfunction. ${ }^{3,4,6,8,15}$ However, the mechanism by which RFP causes mitochondrial dysfunction is unclear. The deleterious effect of RFP was coupled with increased ROS levels. For instance, RFP (150 mg/kg, intragastrically) combined with INH $(75 \mathrm{mg} / \mathrm{kg}$, intragastrically) treatment for 7 days remarkably increased the ROS level in fresh-frozen liver samples from mice, ${ }^{4}$ which was consistent with in vitro findings of the present study and others. ${ }^{16}$ The ROS production partly led to mitochondrial fragmentation. Intriguingly, the loss of Drp1 significantly reduced the ROS level in kidneys and human bronchial epithelial cells upon exposure to injury factors. ${ }^{7,17}$

ROS overproduction, defective ATP synthesis, and reduced mitochondrial membrane potential frequently occur together, and the occurrence of any of these leads to the other two, 
A

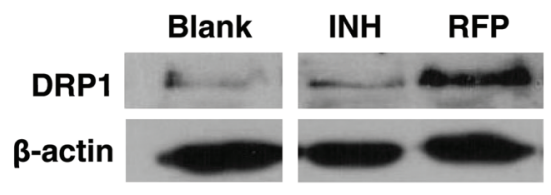

B Tom20
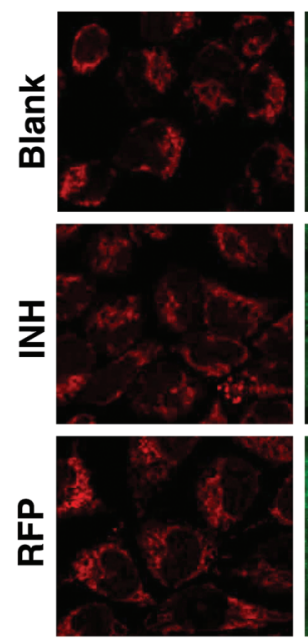

p-DRP1
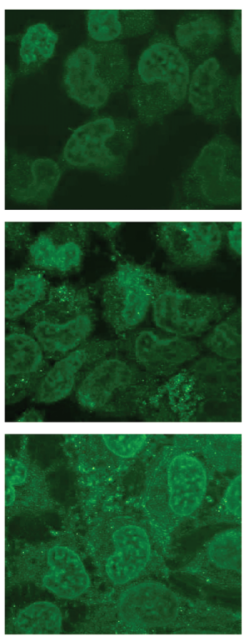

DAPI
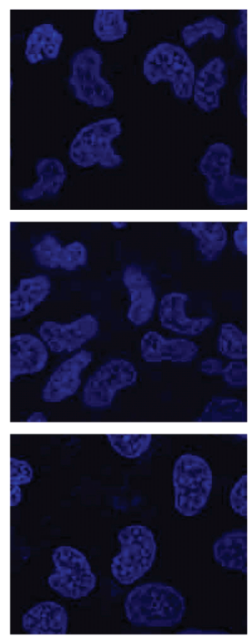

merge
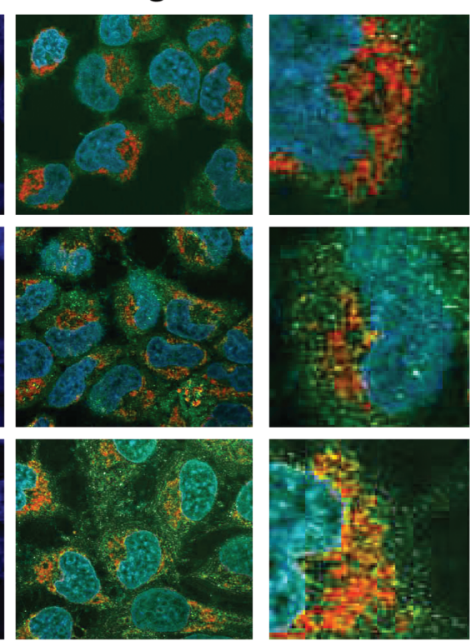
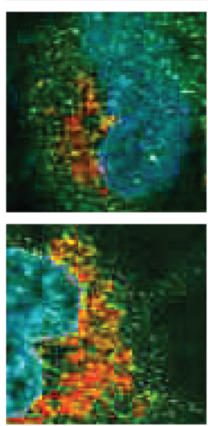

Fig. 3. Effect of RFP and INH on the expression and activation of Drp1 in QSG7701 cells. (A) Western blot analysis showed that RFP obviously increased the expression of DRP1 compared with INH and control. (B) Representative immunofluorescence of Tom 20 (red) and P-Drp1 $1^{\text {s616 }}$ (green) in cells $72 \mathrm{~h}$ after stimulation. $\mathrm{p}$-Drp1 ${ }^{\mathrm{S} 16}$ was primarily localized to mitochondria in cells after RFP exposure compared with INH and control using the confocal imaging analysis.

subsequently establishing a vicious cycle indicating mitochondrial dysfunction. ${ }^{18}$ These are usually associated with changes in mitochondrial morphology, commonly involving disordered mitochondrial dynamics, such as the excessive activation of Drp1. The current report describes confirmatory experiments showing that Drp1 overexpression and activation directly exacerbated hepatocellular apoptosis, while others have shown that manipulation of the translocation and activation of Drp1 significantly reduced hepatocytes apoptosis. ${ }^{19}$ It's also been shown by others that small interfering RNAs' inhibition of Drp1 can significantly alleviate the loss of mitochondrial membrane potential, contributing to mitochondrial death pathways in HT-22 cells. ${ }^{20}$ These results clearly demonstrate that excessive activation of DRP1 and increase of intracellular ROS are in an inescapable network of mutuality. ${ }^{15}$

These finding described herein for RFP- and INH-induced liver injury still need to be investigated in animal experiments, and it will be necessary to explore the origination of the mitochondrial fragments. In conclusion, hepatotoxic effects induced by RFP may be associated with specific Drp1 activation, although the mechanism is not yet clear. The findings to date suggest potential treatment avenues for Drp1-mediated diseases characterized by excessive mitochondrial fission for RFP-induced liver injury. ${ }^{21-23}$

\section{Acknowledgments}

This study was supported by the TCM basis Building Program of State Administration of Traditional Medicine of the People's Republic of China (No. JDZX2015189) and Sanming Project of
Medicine in Shenzhen (SZSM201612014). The authors thank Dr. Xinqiang Xiao for technical assistance in this work.

\section{Conflict of interest}

The authors have no conflict of interests related to this publication.

\section{Author contributions}

Conceived and designed the study (JC), provided the original data (FL), analyzed the results and wrote the manuscript (JZ), provided assistance with data collection (YL), reviewed and edited the manuscript (KS), contributed equally to this paper $(\mathrm{FL}, \mathrm{JZ})$.

\section{References}

[1] World Health Organization. Global tuberculosis report 2016 Available from: http://apps.who.int/iris/bitstream/handle/10665/250441/9789241565394eng.pdf; jsessionid=03BBEC4A2A5A42BA560F37F30EC4D364? sequence $=1$.

[2] Kumar R, Shalimar, Bhatia V, Khanal S, Sreenivas V, Gupta SD, et al. Antituberculosis therapy-induced acute liver failure: magnitude, profile, prognosis, and predictors of outcome. Hepatology 2010;51:1665-1674 doi: $10.1002 /$ hep. 23534

[3] Chowdhury A, Santra A, Bhattacharjee K, Ghatak S, Saha DR, Dhali GK. Mitochondrial oxidative stress and permeability transition in isoniazid and rifampicin induced liver injury in mice. J Hepatol 2006;45:117-126. doi: 10.1016/j.jhep.2006.01.027.

[4] Enriquez-Cortina C, Almonte-Becerril M, Clavijo-Cornejo D, PalestinoDomínguez M, Bello-Monroy O, Nuño $\mathrm{N}$, et al. Hepatocyte growth factor protects against isoniazid/rifampicin-induced oxidative liver damage. Toxicol Sci 2013;135:26-36. doi: 10.1093/toxsci/kft134. 


\section{Li F. et al: Rifampicin induced mitochondria damage}

[5] Awodele O, Akintonwa A, Osunkalu VO, Coker HA. Modulatory activity of antioxidants against the toxicity of Rifampicin in vivo. Rev Inst Med Trop Sao Paulo 2010;52:43-46. doi: 10.1590/S0036-46652010000100007.

[6] Cao J, Mi Y, Shi C, Bian Y, Huang $C$, Ye Z, et al. First-line anti-tuberculosis drugs induce hepatotoxicity: A novel mechanism based on a urinary metabolomics platform. Biochem Biophys Res Commun 2018;497:485-491. doi: 10.1016/j.bbrc.2018.02.030.

[7] Hara H, Araya J, Ito S, Kobayashi K, Takasaka N, Yoshii Y, et al. Mitochondrial fragmentation in cigarette smoke-induced bronchial epithelial cell senescence. Am J Physiol Lung Cell Mol Physiol 2013;305:L737-L746. doi: 10. 1152/ajplung.00146.2013.

[8] Erokhina MV, Kurynina AV, Onishchenko GE. Mitochondria are targets for the antituberculosis drug rifampicin in cultured epithelial cells. Biochemistry (Mosc) 2013;78:1155-1163. doi: 10.1134/S0006297913100106.

[9] Knott AB, Perkins G, Schwarzenbacher R, Bossy-Wetzel E. Mitochondrial fragmentation in neurodegeneration. Nat Rev Neurosci 2008;9:505-518. doi: $10.1038 / \mathrm{nrn} 2417$

[10] Biczo G, Vegh ET, Shalbueva N, Mareninova OA, Elperin J, Lotshaw E, et al. Mitochondrial dysfunction, through impaired autophagy, leads to endoplasmic reticulum stress, deregulated lipid metabolism, and pancreatitis in animal models. Gastroenterology 2018;154:689-703. doi: 10.1053/j. gastro.2017.10.012

[11] Tian L, Neuber-Hess M, Mewburn J, Dasgupta A, Dunham-Snary K, Wu D et al. Ischemia-induced Drp1 and Fis1-mediated mitochondrial fission and right ventricular dysfunction in pulmonary hypertension. J Mol Med (Berl) 2017;95:381-393. doi: 10.1007/s00109-017-1522-8.

[12] Yuan $Y$, Zhang A, Qi J, Wang $H$, Liu X, Zhao $M$, et al. p53/Drp1-dependent mitochondrial fission mediates aldosterone-induced podocyte injury and mitochondrial dysfunction. Am J Physiol Renal Physiol 2018;314:F798F808. doi: 10.1152/ajprenal.00055.2017.

[13] Lee KK, Boelsterli UA. Bypassing the compromised mitochondrial electron transport with methylene blue alleviates efavirenz/isoniazid-induced oxidant stress and mitochondria-mediated cell death in mouse hepatocytes. Redox Biol 2014;2:599-609. doi: 10.1016/j.redox.2014.03.003.
[14] Zhang T, Ikejima $T$, Li L, Wu R, Yuan X, Zhao J, et al. Impairment of mitochondrial biogenesis and dynamics involved in isoniazid-induced apoptosis of HepG2 cells was alleviated by p38 MAPK pathway. Front Pharmacol 2017;8: 753. doi: 10.3389/fphar.2017.00753.

[15] Rogers MA, Maldonado N, Hutcheson JD, Goettsch C, Goto S, Yamada I, et al. Dynamin-related protein 1 inhibition attenuates cardiovascular calcification in the presence of oxidative stress. Circ Res 2017;121:220-233. doi: 10. 1161/CIRCRESAHA.116.310293.

[16] Darvin SS, Esakkimuthu S, Toppo E, Balakrishna K, Paulraj MG, Pandikumar $\mathrm{P}$, et al. Hepatoprotective effect of lawsone on rifampicin-isoniazid induced hepatotoxicity in in vitro and in vivo models. Environ Toxicol Pharmacol 2018;61:87-94. doi: 10.1016/j.etap.2018.05.006.

[17] Perry HM, Huang L, Wilson RJ, Bajwa A, Sesaki H, Yan Z, et al. Dynaminrelated protein 1 deficiency promotes recovery from AKI. J Am Soc Nephrol 2018;29:194-206. doi: 10.1681/ASN.2017060659.

[18] Smith RA, Hartley RC, Cochemé HM, Murphy MP. Mitochondrial pharmacology. Trends Pharmacol Sci 2012;33:341-352. doi: 10.1016/j.tips.2012.03.010.

[19] Zhang C, Huang J, An W. Hepatic stimulator substance resists hepatic ische$\mathrm{mia} /$ reperfusion injury by regulating Drp1 translocation and activation. Hepatology 2017;66:1989-2001. doi: 10.1002/hep.29326.

[20] Grohm J, Kim SW, Mamrak U, Tobaben S, Cassidy-Stone A, Nunnari J, et al. Inhibition of Drp1 provides neuroprotection in vitro and in vivo. Cell Death Differ 2012;19:1446-1458. doi: 10.1038/cdd.2012.18.

[21] Wang Q, Zhang M, Torres G, Wu S, Ouyang C, Xie Z, et al. Metformin suppresses diabetes-accelerated atherosclerosis via the inhibition of Drp1mediated mitochondrial fission. Diabetes 2017;66:193-205. doi: 10 . 2337/db16-0915.

[22] Luo F, Herrup K, Qi X, Yang Y. Inhibition of Drp1 hyper-activation is protective in animal models of experimental multiple sclerosis. Exp Neurol 2017;292: 21-34. doi: 10.1016/j.expneurol.2017.02.015.

[23] Yang $X$, Wang $H$, Ni HM, Xiong $A$, Wang $Z$, Sesaki $H$, et al. Inhibition of Drp1 protects against senecionine-induced mitochondria-mediated apoptosis in primary hepatocytes and in mice. Redox Biol 2017;12:264-273. doi: 10. 1016/j.redox.2017.02.020 